\title{
Leveraging an Open Source VPN Technology to End User
}

\author{
Farok Azmat \\ Faculty of Information Technology and Quantitative Sciences \\ MARA University of Technology (UiTM) \\ 40450 Shah Alam, Selangor, MALAYSIA \\ Tel: 60-3-5521-1148 E-mail: farok@tmsk.uitm.edu.my \\ Md Ikhwan Hamdan \\ Faculty of Information Technology and Quantitative Sciences \\ MARA University of Technology (UiTM) \\ 40450 Shah Alam, Selangor, MALAYSIA
}

Tel: 60-3-5521-1148 E-mail: zegxro@gmail.com

Hj. Kamaruzaman Jusoff (Corresponding author)

Faculty of Forestry, Universiti Putra Malaysia, 43400 Serdang, Selangor, Malaysia

Tel: 60-3-8946-7176 E-mail: kjusoff@yahoo.com

\begin{abstract}
Virtual Private Network (VPN) can be implemented by corporate and public user in a few methods. The methods included proprietary or open source solutions. It has shown great potential due to its encrypted traffic flow which gives it a secure environment. With a good reception of internet in Malaysia it is unfortunate that the major Internet Service Providers (ISPs) have imposed packet filtering. In effect they have throttled the fast broadband to become a slow medium. As a result it gives a disadvantage to active end user. To overcome an ISP packet filtering which slows down internet traffic, this paper intends to study and provide a sample of VPN solution service to end user to improve the current problem.
\end{abstract}

Keywords: BitTorrent, Broadband, File sharing, ISP throttling, Open Source, OpenVPN, P2P, VPN

\section{Introduction}

The Peer-to-Peer (P2P) network is evolving. (Normalkid, 2003) The first generation started by Napster where a client connect to a server to make a search and later downloading is done between peer and peer without server intervention.

The second generation is where peer and peer connect together directly without involving a central server. Therefore there is no particular center point to track this network. It is truly distributed network.

The third generation of P2P improves further in that any client no matter it is Kazaa, Grokster, Morpheus etc although they are different clients they access the exact same file throughout the internet. The enhancement made by this FastTrack protocol is that it includes supernode and spawning which improve searches and download speed.

The fourth generation is dominated by BitTorrent where big files are divided into small pieces of $64 \mathrm{kB}$ until $4 \mathrm{mB}$ where it does not implied much internet bandwidth to the seeder. Files are shared between peers. The pieces may not be in sequence.

\section{Challenges of ISP}

With the new phenomenon of file sharing where upstream and downstream traffic have increased ISP has to look into its broadband infrastructure. Most of the existing ISP is not optimized for "broadband aware" traffic. (Cisco system, undated) When this fact is not observed ultimately it will cause network congestion and performance drops. It is also observed that the common pitfall of ISP when handling the increase in traffic by file sharing is caused by:

(1) The lack of physical attributes of its cable infrastructure. To withstand the new environment to sustain the increase 
in upstream network traffic it needs a high speed data quality cables such as fiber optic nodes.

(2) It also noted that file sharing traffic requires expensive network access point especially on the international link not so to the local network.

With the above facts, it also suggested that the ISP can no longer ignore the current traffic patent used by users. Consequently ISP should start looking at planning and infrastructure upgrade.

\section{Bandwidth throttling}

It is found that $35 \%$ of all internet traffic is used by BitTorrent while the rest are used to distribute all matters of files, distributions of new application and system backup. (Broadbandreports.com, 2004)

Due to the P2P activities most universities have quietly imposed packet filtering using solution such as Ellacoya switch which has captured about US\$200 million market. Other ISP such as Shaw Network (Canada) has also used the same tactic to regain some of their "lost" bandwidth.

\section{Bandwidth throttling in Malaysia}

As in Malaysia the first encounter of packet filtering is felt when Maxis imposed in its "Terms and Conditions" a statement which states that an automatic disconnection to the customer's internet connection is imposed after a certain period of inactivity. (Asohan, 2007) The period may vary from 20 to 30 minute of inactivity. Another means of blocking by Maxis is to limit customer total usage per month NOT exceeding 3GB of data volume per customer.

It is also believed that another ISP, TM Net the provider of Streamyx broadband service has also imposed a similar tactic since February 2006 but it was greatly felt sometimes mid-September 2006. When customer complaint about the slowness of Streamyx TM Net conceded that $20 \%$ of its customer uses P2P services and causes the maximum utilization of its network capacity but it still hold back its upgrade to its network infrastructure to a later requirement. (Teoh, 2006)

\section{Managing heavy traffic}

According to the Cisco systems white paper, (Cisco system, undated) ISP should realize that there are factors to be taken into account when considering planning network bandwidth. It gives the following facts and suggestions as in Table I.

\section{<<Table 1. Factor for planning network bandwidth (Source: Cisco systems white paper)>>}

The paper also highlighted that the present major selling attraction to ISP is the broadband. It should also realize that the current technology has deviated from traditional "client-server" model to a multiple "client-server" model as shown by most sharing program protocols. They are: KaZaA, Gnutella, Winny, WinMX, eDonkey, BitTorrent, DirectConnect, Manolito, Kuro, Soulseek, Filetopia, iTunes, Napster, Waste, Mute, Share etc.

Moreover, the present internet technology has advanced from its traditional 'client-server' model which is also called Content Delivery Networks (CDN) and it has up-grown to a hybrid CDN which consumes more bandwidth. With this hybrid CDN as shown in the file sharing technology and high quality video delivering, ISP should take these into account when designing its infrastructure. (Klinker, 2008)

Thus based on the above factors the ISP can easily analyze, manage and implement its infrastructure to its benefits or it's Return on Investment (ROI) will not be achieved.

\section{Practical method of bypassing ISP throttling}

To address the traffic shaping by TM Net Streamyx, one possible solution is to implement OpenVPN technology. There are two components involved. One is the VPN server and the other is the client.

The main question here is where will the VPN server be located?

Basically Figure 1 illustrates the implemented location of VPN server

\section{$<<$ Figure 1. Location of The Implemented VPN Server $>>$}

The Figure 1 above shows the implemented VPN server. It is located before the $10 \mathrm{~Gb}$ International Line backbone to Malaysia (TM, 2007) by hiring a reasonable price at a site located in US. The VPN server is actually on the high speed backbone line. Bear in mind although the server is on the very high speed backbone the user will be expected a fully $1 \mathrm{Mb} / \mathrm{s}$ speed. Thus the effect of using VPN technology:

(1) All applications used by the client will pass through the VPN server.

(2) Due to the unblocked port as granted by the ISP, the speed of transaction is available at a considerably higher speed as compared to the previous one. See Figure 2 and Figure 3 for the comparison between P2P downloading without and with VPN respectively.

\section{$<<$ Figure 2. Downloads Performance without VPN>>}




\section{$<<$ Figure 3. Downloads Performance with VPN $>>$}

The Figure 2 above shows the speed of normal downloading without VPN. It is $9.5 \mathrm{kByte} / \mathrm{s}$. This shows that TMNET is imposing the bandwidth throttling. While Figure 3 shows the downloading speed with VPN. It has improved to $108 \mathrm{kByte} / \mathrm{s}$. Therefore it shows that VPN is a valid method to circumvent the bandwidth throttling done by TMNET Streamyx.

A simple check is required here. (helpwithpcs.com, 2008) If the user bandwidth provided by TMNET Streamyx is $1 \mathrm{Mbit} / \mathrm{s}$ it can be written as:

$S=\frac{B W(\text { bit } / s)}{8}$

Where:

$S=$ speed in Byte/s

$B W($ bit $/ s)=$ bandwidth

In this case applying Equation (1) to the Streamyx bandwidth of 1Mbit/s becomes:

$$
\begin{gathered}
S=\frac{1 M(\mathrm{bit} / \mathrm{s})}{8} \\
S=125 \mathrm{kByte} / \mathrm{s} \#
\end{gathered}
$$

Comparing this ideal case to the speed obtained from the exercise which is $108 \mathrm{kByte} / \mathrm{s}$ the speed is most appreciated.

\section{Simple equations for user}

The download time for any given file size can be determined by a general equation given as below:

$$
T(\mathrm{sec})=\frac{F(\text { MByte } \times 8)}{B W\left(\frac{M b i t}{s}\right)}
$$

Now consider an ideal condition as follows.

Example 1

Let the file size be

$$
F=1 M B y t e
$$

Let the broadband bandwidth be:

$B W=\frac{1 M b i t}{s}$

Note that the bandwidth of the line is also normally known as the throughput of the line.

Then using Equation (2) the time to download a given file is:

$$
\begin{gathered}
T(\mathrm{sec})=\frac{F(M B y t e \times 8)}{B W\left(\frac{M b i t}{s}\right)} \\
T(\mathrm{sec})=\frac{1(M B y t e \times 8)}{1\left(\frac{M b i t}{s}\right)} \\
T(\mathrm{sec})=\frac{8 M b i t}{\frac{1 M b i t}{s}} \\
\underline{T(\mathrm{sec})=8 \mathrm{sec}} \#
\end{gathered}
$$

Therefore the time to download a $1 \mathrm{MByte}$ of data in a $1 \mathrm{Mbit} / \mathrm{s}$ line is in $8 \mathrm{sec}$.

Example 2

Let's consider another example

Let the file size be: 


$$
F=1 G B y t e
$$

Let the broadband bandwidth be:

$$
B W=\frac{1 M \text { bit }}{s}
$$

Then using Equation (2) again the time to download the given file is:

$$
\begin{gathered}
T(\mathrm{sec})=\frac{1(\text { GByte } \times 8)}{1\left(\frac{\text { Mbit }}{\mathrm{s}}\right)} \\
T(\mathrm{sec})=\frac{1\left(10^{9} \text { Byte } \times 8\right)}{1\left(\frac{10^{6} \mathrm{bit}}{\mathrm{s}}\right)} \\
T(\mathrm{sec})=8000 \mathrm{sec} \\
T(\mathrm{~min})=\frac{8000}{60} \\
T(\mathrm{~min})=133.33 \mathrm{sec} \\
T(\mathrm{hr})=\frac{133.33}{60} \\
T(\mathrm{hr})=2.2 \mathrm{hour} \\
\underline{T(\mathrm{hr})=2 \mathrm{hr} 12 \mathrm{~min}} \#
\end{gathered}
$$

Therefore the time to download a 1GByte of data in a 1Mbit/s line is in 2 hour $12 \mathrm{~min}$.

But the above formula is only valid for an ideal download through a 1Mbit/s broadband line. And in actual fact there exist a time interval between when a network station seeking access to a transmission channel and when the access is granted or received. This amount of time elapsed between receiving and retransmitting of the LAN packet is called latency. (Themanualpage.org, 2002)

Latency also depends on the speed of the transmission medium such as the copper wire used, optical fiber or radio wave. It also includes the delay of the transmission devices such as modem and router. Thus a low latency figure indicates high network efficiency.

Therefore in a real case there are a number of factors to be considered such as interference of data which caused delay, the quality of telephone line and distance of user from substation. These factors or latency will degrade the download traffic to $260 \mathrm{~ms}$ or $26 \%$ of the actual speed. (Cheshire, 1996)

From the above argument the real download time in hours considering the worst case of 30\%, the Equation (2) becomes:

$$
T(\mathrm{sec})=\frac{F(\text { MByte } \times 8)}{B W\left(\frac{M b i t}{s}\right)} \times(100-30) \%
$$

Applying the Equation (3) to the result obtained in Example 2 the time of 2 hour 12 min becomes 2 hour 52 min. Thus in real life the download time is much longer then the ideal download time by about $30 \%$.

\section{Conclusion}

This paper has clearly illustrated that VPN is able to circumvent the ISP throttling in Malaysia. The important point to note is that the VPN server should be located on the high bandwidth backbone.

The other advantages gained by end user are:

(1) Encrypted traffic - Nobody can sniff the traffic even the ISP.

(2) Able to surf anonymously - Not to reveal the actual IP address of origin. To be used as if the surfer is from the server site IP.

(3) Able to bypass site which has been blocked by country of origin. 


\section{References}

Asohan, A (2007). Throttling broadband access for all, Available online: http://thestar.com.my/news/story.asp?file=/2007/2/8/focus/16811744\&sec=focus, Downloaded: August 13, 2007

Broadbandreports.com (2004). Shaw Throttling Bit Torrent?, Available online: http://www.broadbandreports.com/shownews/56419, Downloaded: May 272007

Cisco systems (undated), Managing Peer-To-Peer Traffic With Cisco Service Control Technology, White paper, Available online: http://www.cisco.com/en/US/prod/collateral/ps7045/ps6129/ps6133/ps6150/prod_white_paper0900aecd8023500d_ps61 35_Products_White_Paper.html, Downloaded:April 1, 2008

Klinker, E (2008). When should you choose P2P?, Available online: http://www.streamingmedia.com/article.asp?id=10068, Downloaded: April 152008

helpwithpcs.com (2008). Internet Connections explained, A guide to dial-up, ADSL and Cable connections, Available online:

http://www.helpwithpcs.com/internet/internet-connections.htm, Downloaded: 31 March 2008

Normalkid, L.L.C (2003). Peer to Peer (P2P) Introduction and History, Available online: http://www.mac-p2p.com/p2p-history/, Downloaded: May 112008

Teoh, S (2006). TM Net: P2P traffic clogging broadband, Available online: http://star-techcentral.com/tech/story.asp?file=/2006/3/1/technology/13540730\&sec=technology, Downloaded: August 13,2007

Cheshire, S (1996). Latency and the quest for Interactivity, Available online: http://www.stuartcheshire.org/papers/LatencyQuest.ps, Downloaded: 31 March 2008

Themanualpage.org (2002). Glossary - L, Available online: http://www2.themanualpage.org/glossary/glo_l.php3, Downloaded: 31 March 2008

TM News Release (April 2007). TM leads 17 -member Consortium of Telcos to establish Asia-America Gateway (AAG) - The first submarine cable system linking South East Asia directly to USA, Available online: http://www.tm.com.my/about tm/newsroom/2007/070427.htm, Downloaded: Feb 14, 2008

Table 1. Factor for planning network bandwidth (Source: Cisco systems white paper)

\begin{tabular}{|c|l|}
\hline $\begin{array}{c}\text { Parameter } \\
\text { Dpstream / } \\
\begin{array}{c}\text { ratio } \\
\text { ram traffic }\end{array}\end{array}$ & $\begin{array}{l}\text { The amount of upstream and downstream traffic flow should be } \\
\text { tailored according to the correlation to application requirement } \\
\text { according to its users }\end{array}$ \\
\hline $\begin{array}{c}\text { Time of day and } \\
\text { percentage of activity }\end{array}$ & $\begin{array}{l}\text { Based on the users usage there must exist "rush hour" or "peak } \\
\text { hour" during B2B or B2C activities. Most home user may peak at } \\
\text { the evening till night, weekend and holidays }\end{array}$ \\
\hline $\begin{array}{c}\text { Traffic destination and } \\
\text { peering point }\end{array}$ & $\begin{array}{l}\text { Traffic within the local network backbone and international } \\
\text { connection should be reviewed and considered }\end{array}$ \\
\hline $\begin{array}{c}\text { Estimated traffic } \\
\text { volume }\end{array}$ & $\begin{array}{l}\text { Network bandwidth is fixed for all users and a certain over } \\
\text { subscriptions assumption should be planned accordingly to } \\
\text { calculate current network capacity }\end{array}$ \\
\hline
\end{tabular}




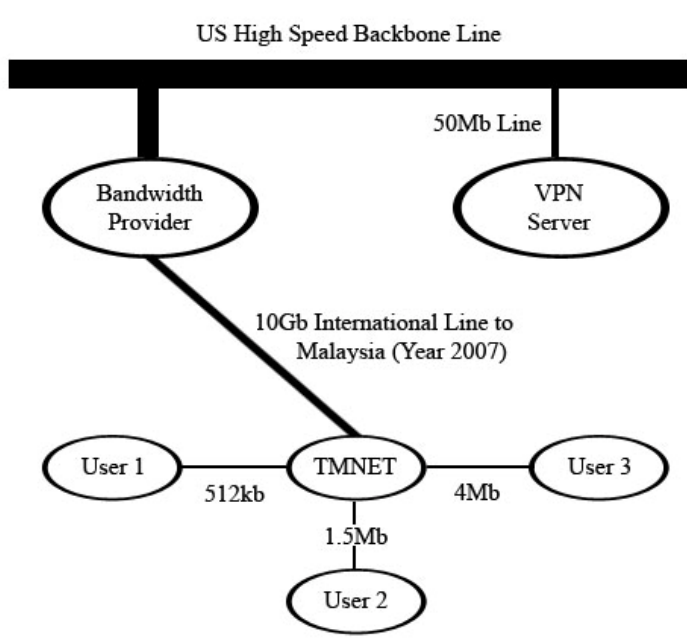

Figure 1. Location of the Implemented VPN Server

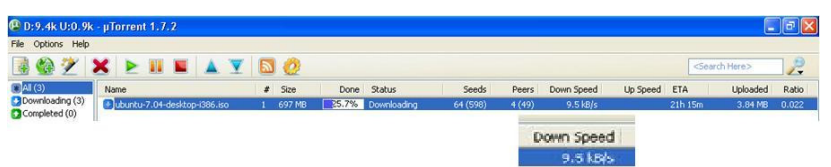

Figure 2. Downloads Performance without VPN

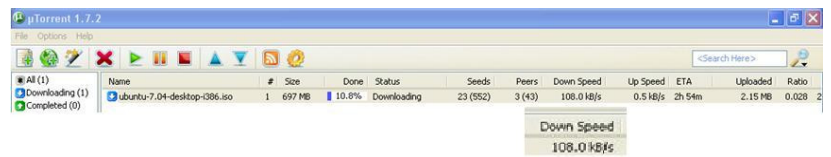

Figure 3. Downloads Performance with VPN 